\title{
Gene therapy for haemophilia: a very modern success story
}

Amit Nathwani

AAV-mediated gene therapy has the potential to be paradigm shifting as a treatment for haemophilia. A single administration of AAV vector can result in safe and consistent long-term expression of transgene ( $>5$ years), reduction in spontaneous bleeding episodes, reduction in clotting factor usage and an improvement in quality of life. There is huge commercial interest in this approach, with the expectation that an AAV gene therapy product for haemophilia B will be licensed by 2020 .

Keywords: gene therapy, AAV, haemophilia, Katharine Dormandy

Gene therapy in haemophilia offers the possibility of a cure; with the continuous endogenous expression of either factor VIII or factor IX at therapeutic levels after a single administration of vector. Haemophilia has been at the forefront of gene therapy because it is extremely wellsuited to this approach:

- Single gene defect

- The therapeutic goal is modest - an increase in plasma factor VIII or FIX levels above 1\% of physiologic levels is sufficient to ameliorate the bleeding phenotype

- Efficacy can be assessed by validated routine laboratory assay

- Tight regulation is not required - a wide range of factor VIII and factor IX levels is likely to be efficacious and non-toxic.

Of the large number of gene transfer vectors available for gene therapy, we focused on the adeno-associated viral (AAV) vector system primarily because of its excellent safety profile. Infections with wild type AAV are common in humans but this does not result in any disease pathology. Additionally, AAV is naturally replication deficient, which adds another layer of safety. Importantly, animal studies (including nonhuman primates) showed that a single administration of AAV results in stable long-term expression of transgenic protein such as FIX for periods that extend to 10 years.

Our approach for gene therapy of haemophilia B with $A A V$ vectors included two important changes from previous approaches with this vector system:

- A more potent vector (self-complementary vector; scAAV2/8-LP1-hFIXco) was developed by modifying the genome in the hope that therapeutic levels of FIX could

Professor Amit Nathwani, director of the Katharine Dormandy Haemophilia Centre, Royal Free Hospital, London

Email: a.nathwani@ucl.ac.uk

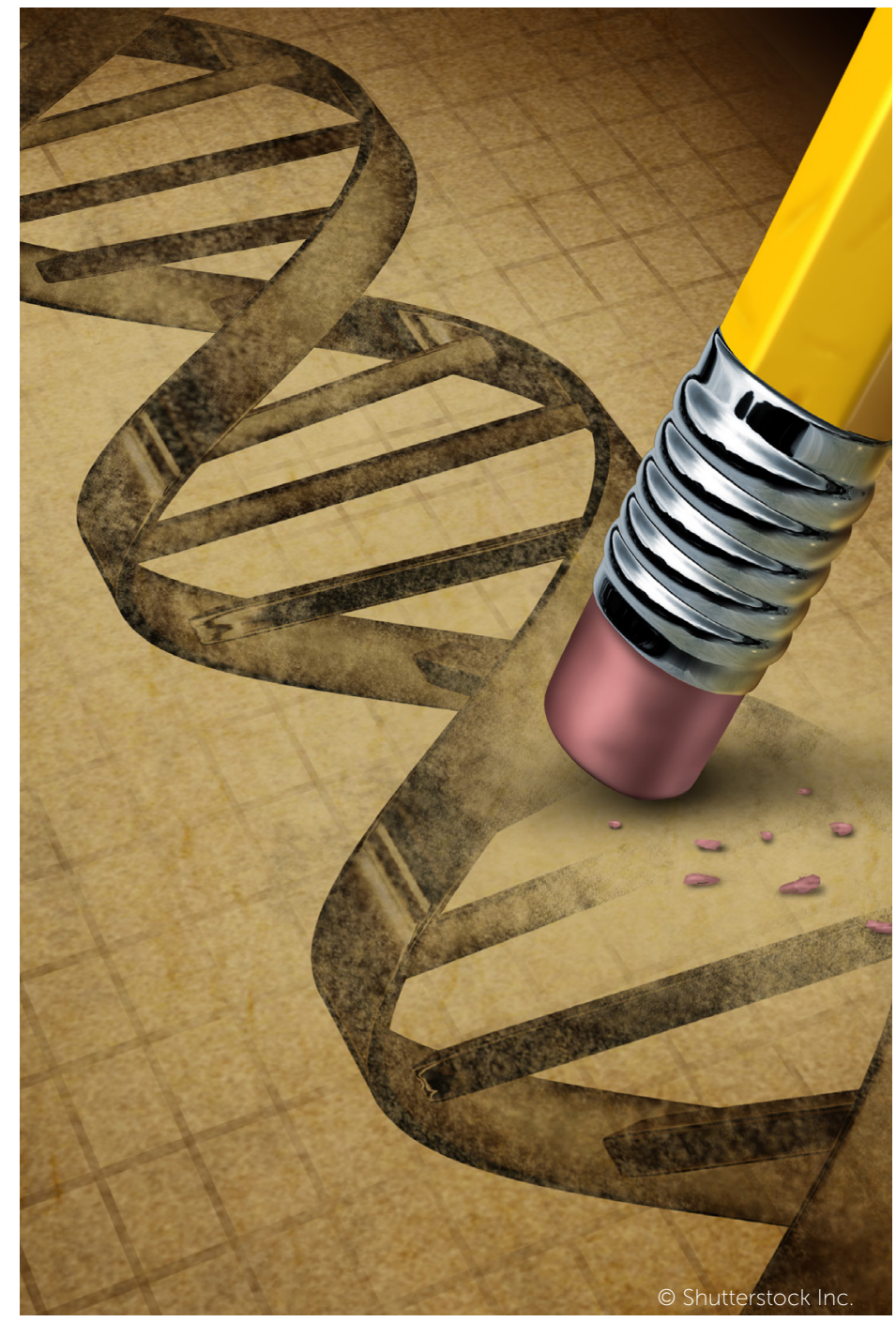

be achieved following a single administration of vector A simple non-invasive route of vector administration, which involved a single bolus infusion of vector into the peripheral vein, was used because of the ability of AAV8 capsid that was used in our study to "home" to the liver and preferentially deliver the gene to hepatocytes, which is where FIX is normally made.

The phase I trial

The objective of our phase I trial was to assess the safety and efficacy of bolus administration of this self-complementary vector into patients with severe haemophilia B (factor IX concentration $<1 \%$ ), without 
upfront immunosuppression at the time of the active administration.

Because we already have very good therapy for haemophilia B, we embarked on an elaborate three-stage informed consent process that involved an assessment by an independent ombudsman: his role was to ensure that all patients were fully aware of the risks of participating [1] The study was a collaboration between the Royal Free Hospital and St Jude's Children's Research Hospital in the United States. The first six patients were recruited at the Royal Free and the patient demographics have been reported [2]. Six subjects with severe haemophilia B (FIX levels $<1 \%$ ) were enrolled to the initial phase, with two subjects recruited sequentially at one of three vector

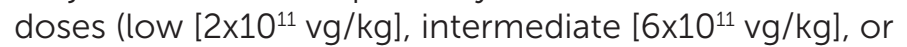
high dose $\left[2 \times 10^{12} \mathrm{vg} / \mathrm{kg}\right]$ ) of scAAV2/8-LP1-hFIXco. Factor IX expression at 1-6\% of normal was established in all six subjects in a dose dependent manner. Asymptomatic, transient elevation of serum liver enzymes, likely a result of an immune response to the AAV8 capsid, was observed in both subjects recruited to the high dose level. Treatment of each with a short course of prednisolone led to rapid normalisation of liver enzymes and maintenance of FIX levels in the $2-4 \%$ range.

FIX expression has remained relatively stable over a period of at least 5 years with a follow-up period that ranges from 2.5-5.5 years.

Based on these promising data we recruited four new subjects to the high dose level so that we could better understand the key AAV-related adverse event, which was the asymptomatic rise in liver enzymes associated with a decline in FIX levels observed in the two subjects previously treated at the high dose level. Expansion of the high dose cohort showed that this adverse event was relatively common, occurring in four of the total of six subjects treated at the high dose level. Remarkably the rise in liver enzymes occurred at a consistent time point of between 7-10 weeks after gene transfer, thus defining the critical period of monitoring and pharmacological intervention. Once again a short course of prednisolone was effective at limiting the hepatocellular toxicity as well as preserving expression of transgenic FIX, especially when commenced early. Extended follow-up of all the high dose patients showed that the transaminitis does not re-occur at later time points, following cessation of prednisolone therapy.

Importantly FIX activity was remarkably consistent within the six patients in the high dose cohort with an average steady-state level of $5.1 \pm 1.7 \%$. As a consequence, four of the seven subjects who were on prophylaxis prior to gene therapy where able to stop regular FIX replacement therapy, while the others were able to increase the interval between prophylactic FIX infusions. The overall reduction in FIX concentrate usage over the duration of the study in the 10 subjects recruited has been $>4$ million units thus far, resulting in a financial saving that is in excess of $£ 2.5$ million. Despite the reduction in FIX usage, the average annualised bleeding rate was consistently lower after gene transfer despite higher overall activity levels reported by all subjects which included participation in physical sporting activities that had previously been associated with bleeding.

Therefore, our gene therapy approach, even with its risk of transient asymptomatic hepatic inflammation, has the potential to convert the severe haemophilia B bleeding phenotype into a mild form of the disease or to reverse it entirely through durable FIX expression and longlasting amelioration of the bleeding diathesis following a single systemic administration of vector. The impact of gene therapy on the majority of the participants has been life-changing. Hence, AAV mediated gene transfer has the potential to change the treatment paradigm for haemophilia B.

\section{Where are we now?}

Our data has attracted huge interest from the pharmaceutical sectors, including many of the companies that are already in the haemophilia sector. This is highly encouraging as investment from the industry is required to accelerate the development of the gene therapy approach so that it becomes licenced and available to the wider haemophilia community.

We have recently started explored the possibility of using the same AAV platform for haemophilia A, which is five times more common than haemophilia B. The use of AAV vectors for haemophilia A gene therapy, however, poses two new challenges due to the distinct molecular and biochemical properties of human FVIII, the co-factor that is malfunctional in these patients. Firstly, when compared to other proteins of similar size, expression of human FVIII is highly inefficient. Additionally, the FVIII coding sequence spans $7.0 \mathrm{~kb}$, which far exceeds the normal packaging capacity of AAV vectors of $4.6 \mathrm{~kb}$. We have, therefore, developed an AAV based gene transfer approach that addresses both the size constrains and inefficient FVIII expression. Expression of human FVIII was improved 10fold by reorganisation of the wild type cDNA of human FVIII according to the codon usage of highly expressed human genes. Expression from B-domain deleted codon optimised FVIII molecule was further enhanced by the inclusion of a 17 amino-acid peptide that contains the six $\mathrm{N}$-linked glycosylation signals from the B domain required for efficient cellular processing. These changes have resulted in a novel $5.2 \mathrm{~kb}$ AAV expression cassette (AAV-HLP-codop-hFVIII-V3) that is efficiently packaged into recombinant $A A V$ vectors and capable of mediating supraphysiological level of FVIII expression in animal models over the same dose range of AAV8 that proved to be efficacious in subjects with haemophilia B. A new gene therapy trial for patients with severe Haemophilia A 


\section{Amit Nathwani}

Professor Amit C Nathwani is the Director of the Katharine Dormandy Haemophilia Centre at the Royal Free Hospital and a Senior NIHR Investigator. He is also Professor of Haematology at UCL. He graduated in Medicine from the University of Aberdeen in 1984. His PhD was on the regulation of the tissue factor gene. In 1997 he moved to St Jude Children's Research Hospital, Memphis, Tennessee, USA, to work with Dr Arthur Nienhuis on adeno-associated virus mediated gene transfer, which is where he started his pioneering work on gene therapy for haemophilia B. In 2001 he returned to University College London as a Senior Lecturer in Haematology and a Consultant to the National Blood Services in the UK. Professor Nathwani's team of clinical and non-clinical scientists and students are currently engaged in a diverse range of translational research including gene therapy for haemophilia A, congenital bleeding disorder, chronic lymphocytic leukaemia and age-related macular degeneration.

will commence at the Katharine Dormandy Haemophilia Centre for patients with in the first quarter of 2016 , marking a new era in the treatment of haemophilia $A$, that has the potential of a cure.

\section{Acknowledgement}

The author would like to thank the patients of the Royal Free Hospital. Their bravery permitted us to perform a proof-of-concept, first-in-man study without any expectation of benefit. In addition, thanks to the staff of UCL/Royal Free Hospital (particularly Edward Tuddenham)، and of the St Jude Children's Research Hospital (particularly Andrew Davidoff).

\section{Disclosures}

The scientific meeting on which this issue of The Journal of Haemophilia Practice is based was sponsored by Baxter, Bayer, CSL Behring, Grifols, Novo Nordisk, Sobi, Pfizer, BPL and Werfen. Editorial support for the article was provided by the publisher.

\section{References}

1. Morris J. Patient advocacy helps patients weigh up gene therapy trial risk/benefits. J Haem Pract 2015; 2(1): 6-8. doi: 10.17225/jhp.00040. Avaialble at http://www. haemjournal.com/external/articles/doi/10.17225/jhp.00040

2. Nathwani AC, Tuddenham EG, Rangarajan S, et al. Adenovirus-associated virus vector-mediated gene transfer in hemophilia B. N Engl J Med 2011; 365(25): 2357-

65. doi: 10.1056/NEJMoa1108046.

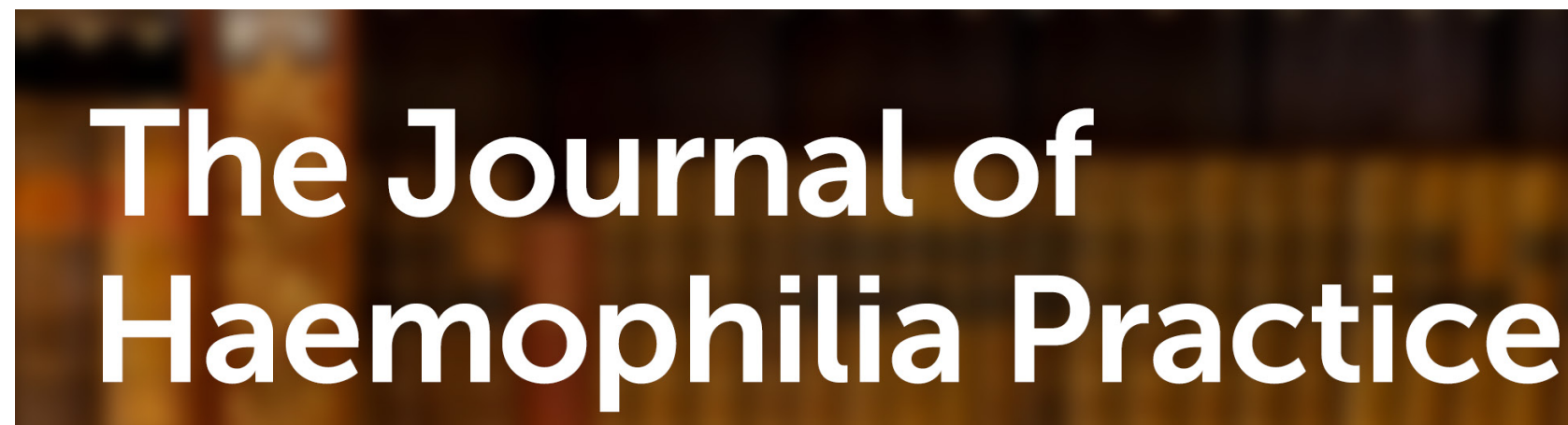

\section{An open-access journal for sharing experience in the care of people with bleeding disorders}

